\title{
Fast Analysis for Aperture Efficiency and Radiation Patterns of Reflectarray Antennas
}

\author{
Yan Qu, Chenjiang Guo, Hua Guo and Jun Ding \\ School of Electronics and Information, Northwestern Polytechnical University, Xi'an \\ 710129, China \\ quyan0908@gmail.com
}

\begin{abstract}
In the design procedure of the reflectarray antennas, the fast analysis of the aperture efficiency and radiation patterns is necessary before manufacture to forecast the antennas performance proximately. The fast calculation of the aperture efficiency and radiation patterns of reflectarray antennas is presented in this paper. A more general approach is introduced to calculate the spillover efficiency of a reflectarray. Meanwhile, the taper efficiency of the reflect-array is calculated with a unified set of equations. Two different fast approaches of the radiation patterns analysis-array theory and aperture field are described, and the discrete Fourier transform is applied to speed up the computational speed. On the basis of these derivations, numerical results of a Ku band reflectarray with offset feed are presented to show the performance of these approaches, and to provide the guidelines for designing and analyzing reflect-array antennas.
\end{abstract}

Keywords: spillover efficiency, taper efficiency, radiation patterns, reflectarray

\section{Introduction}

In the last decade, the reflectarray antennas have been received notable attentions because it combines the favorable features of the reflectors and phased arrays [1-3]. The phase of each reflectarray element can be individually controlled to achieve the specific radiation patterns, whereas the spatial feeding eliminates the energy loss and the design complexity of a feeding network. As such, the reflectarray antennas become a new competitive candidate for highgain antennas.

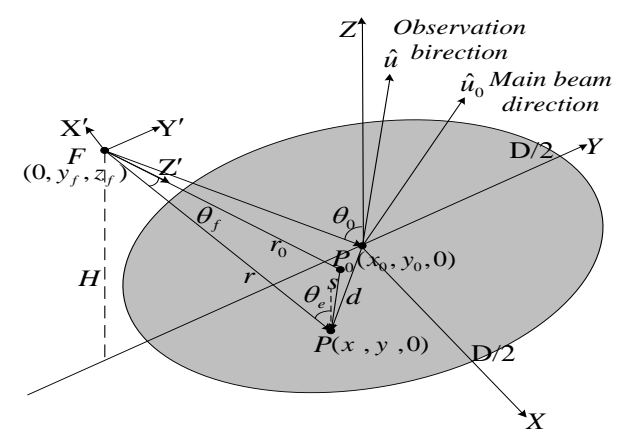

Figure 1. Coordinate System and Configuration Parameters of a General Reflectarray System 
Similar to the design of the conventional reflectors [4], the aperture efficiency and radiation characteristics need to be predicted in the initial reflect-array design procedure to evaluate the antennas performances. Considering the electrically large size of the reflect-array antennas' aperture, combined with hundreds of elements with dimensions smaller than a wavelength, a full wave simulation demands a significantly high computational times and large resources. Therefore, the time-efficient and relatively accurate approximated method should be introduced.

In this paper, the aperture efficiency and radiation characteristics of a general reflectarray system characterized by a group of configuration parameters, as showed in figure 1, will be investigated by using the fast approximated approach. A more general approach to calculate the aperture efficiency is introduced, which can help us to calculate the spillover efficiency without determination of solid angle with respect to the feed [1,6]. Two classical approaches--array theory and aperture field $[1,5]$ for reflect-array radiation patterns analysis are reviewed. A comprehensive derivation will be presented on how the aperture efficiency and radiation patterns are determined to help antenna engineers for the program development of these methods. Numerical results are presented for the off-set feed reflect-array configuration, using these approaches.

\section{Model of Analysis}

Table 1. Primary Geometric Parameters

\begin{tabular}{ll}
\hline Parameter & Formula \\
\hline Feed location & $F\left(0, y_{f}, z_{f}\right)$ or $\left(0,-H \tan \theta_{0}, H\right)$ \\
Feed beam point(FBP) & $P_{0}\left(x_{0}, y_{0}, 0\right)$ \\
Element location & $P_{m n}\left(x_{m n}, y_{m n}, 0\right)$ \\
Distance between the feed and & $r_{0}=\sqrt{x_{0}^{2}+\left(y_{0}-y_{f}\right)^{2}+z_{f}^{2}}$ \\
FBP & $r_{m n}=\sqrt{x_{m n}^{2}+\left(y_{m n}-y_{f}\right)^{2}+z_{f}^{2}}$ \\
Distance between the feed and & $\begin{array}{l}\text { element } \\
\text { Unit vector from feed to }\end{array}$ \\
element & $\bar{r}_{m n}=\left(x_{m n} \hat{x}+\left(y_{m n}-y_{f}\right) \hat{y}+\left(-z_{f}\right) \hat{z}\right) / \sqrt{x_{m n}^{2}+\left(y_{m n}-y_{f}\right)^{2}+z_{f}^{2}}$ \\
Distance between element and & $s_{m n}=\sqrt{\left(x_{m n}-x_{0}\right)^{2}+\left(y_{m n}-y_{0}\right)^{2}}$ \\
FBP & $\cos \theta_{f}=\left(r_{0}^{2}+r_{m n}^{2}+s_{m n}^{2}\right) / 2 r_{0} r_{m n}$ \\
Feed pattern parameter & $\cos \theta_{e}=z_{f} / r_{m n}$ \\
Element pattern parameter & $\hat{u}_{0}=\hat{x} \sin \theta_{b} \cos \phi_{b}+\hat{y} \sin \theta_{b} \sin \phi_{b}+\hat{z} \cos \theta_{b}$ \\
Unit vector of main beam & $\hat{u}=\hat{x} \sin \theta \cos \phi+\hat{y} \sin \theta \sin \phi+\hat{z} \cos \theta$ \\
direction & $\vec{d}_{m n}=\hat{x} x_{m n}+\hat{y} y_{m n}$ \\
Unit vector of observation & direction \\
Vector from origin to element &
\end{tabular}

Figure 1 illustrates the Cartesian coordinates systems established for reflect-array analysis. The coordinate $x y z\{\hat{c}\}$ is the reflectarray coordinate, whose origin is located at the center of the reflectarray aperture and the $x$ and $y$ axes are set on the aperture plane. The coordinate $x^{\prime} y^{\prime} z^{\prime}\left\{\hat{c}^{\prime}\right\}$ is the feed coordinate, whose origin is located at the 
phase center of the feed horn and $z^{\prime}$ axes points to a point $P_{0}$ on the array plane. The reflectarray aperture is illuminated by a feed horn whose pattern function is approximated by a cosine $q$ model and phase center is located at $F$ [1]. For the reflectarray element located at $P$, its pattern is also approximated by a cosine $q$ model [6]. The Observation direction is in $\hat{u}$ with the spherical angular $(\theta, \phi)$, and the main beam directs in $\hat{u}_{0}$ direction with the spherical angular $\left(\theta_{b}, \phi_{b}\right)$.To facilitate the following derivation and analysis, some primary geometric parameters involved are listed in Table 1.

\subsection{Derivation of Aperture Efficiency}

To proximately analyzing efficiency characteristic of reflectarray antennas, the aperture efficiency discussed here does not include these efficiency factors associated with the mismatch loss, polarization loss, reflectarray element loss, and feed loss, which are involved in a full wave simulation. Such that, only two major factors, the spillover efficiency and the taper efficiency, are studied to predict the efficiency characteristic of reflectarray antennas. The aperture efficiency is defined as their product:

$$
\eta_{a}=\eta_{s} \eta_{t}
$$

With the given pattern function model of the feed horn and element, their normalized power pattern [6] can be expressed:

$$
\begin{aligned}
& U_{f}(\theta, \phi)= \begin{cases}\left(\cos \theta_{f}\right)^{2 q_{f}} & \left(0 \leq \theta_{f} \leq \pi / 2\right) \\
0 & \text { elsewhere }\end{cases} \\
& U_{e}(\theta, \phi)= \begin{cases}\left(\cos \theta_{e}\right)^{2 q_{e}} & \left(0 \leq \theta_{e} \leq \pi / 2\right) \\
0 & \text { elsewhere }\end{cases}
\end{aligned}
$$

Where $q_{f}$ and $q_{e}$ are the pattern factors of the feed horn and element, respectively. The value of $q_{f}$ can be determined from the measured or simulated data of the feed horn, which commonly has value from 5 to 8 , and the $q_{e}$ can be determined by the directivity of element, which often has a smaller value such as 1 .

\subsubsection{The Spillover Efficiency}

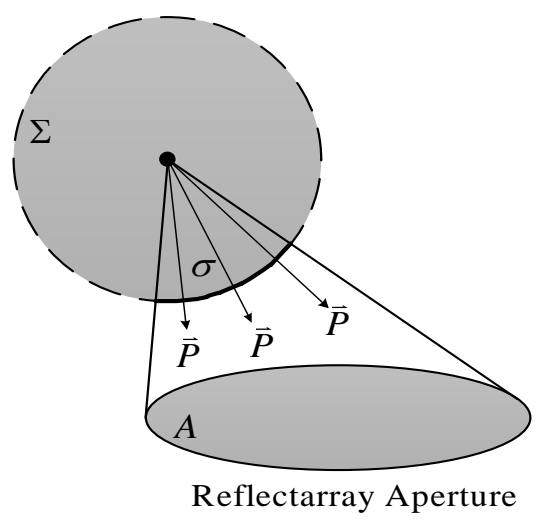

Figure 2. Reflectarray Geometry for Spillover Efficiency Analysis 
For the reflectarray antennas, the spillover efficiency $\eta_{s}$ is defined as the percentage of the radiated power from the feed that is intercepted by the reflectarray aperture [4]. As illustrated in Figure 2, the evaluation of $\eta_{s}$ is through the following equation:

$$
\eta_{s}=\frac{\iint_{\sigma} \vec{P}(\vec{r}) \square d \vec{s}}{\iint_{\Sigma} \vec{P}(\vec{r}) \square d \vec{s}}
$$

Where $\vec{P}(\vec{r})$ is the Poynting vector of the feed? The method to calculate the spillover efficiency through equation (4) is called original method.

Practically, only the dimensions of the reflectarray aperture are known [6], hence it is necessary to determine the boundary of $\sigma$ in terms of the spherical coordinates of the feed. In most cases, except for center feed, it is not easy to determine the boundary of $\sigma$ through reflectarray aperture. Thus, calculating the spillover efficiency for offset feed cases becomes difficult. However, noting that the reflectarray aperture and spherical surface portion $\sigma$ share the same solid angle with respect to the feed, the integral in the numerator of equation (4) can be performed over the reflectarray aperture instead of on the spherical surface portion $\sigma$, the equation (4) can be rewritten:

$$
\eta_{s}=\frac{\iint_{A} \vec{P}(\vec{r}) \square d \vec{s}}{\iint_{\Sigma} \vec{P}(\vec{r}) \square d \vec{s}}
$$

The method to calculate the spillover efficiency through equation (5) is called new method.

With the known normalized power pattern, the Poynting vector of the feed horn can be written:

$$
\vec{P}(\vec{r})=\hat{r} \frac{\left(\cos \theta_{f}\right)^{2 q_{f}}}{r^{2}}\left(0 \leq \theta_{f} \leq \frac{\pi}{2}\right)
$$

Accordingly, the denominator in equation (4) or (5) can be calculated analytically:

$$
\begin{aligned}
I_{d} & =\int_{0}^{2 \pi / 2} \int_{0}^{\pi / 2}\left(\hat{r} \frac{\left(\cos \theta_{f}\right)^{2 q_{f}}}{r^{2}}\right)\left(\hat{r} r^{2} \sin \theta_{f}\right) d \theta_{f} d \phi_{f} \\
& =\int_{0}^{2 \pi / 2} \int_{0}^{\pi / 2}\left(\cos \theta_{f}\right)^{2 q_{f}} \sin \theta_{f} d \theta_{f} d \phi_{f}=\frac{2 \pi}{2 q_{f}+1}
\end{aligned}
$$

To calculate the numerator in equation (5), the Poynting vector should be rewritten in the Cartesian coordinates using the configuration parameters in Table 1as follow:

$$
\vec{P}(\vec{r})=\frac{1}{r^{3}}\left(\frac{r_{0}^{2}+r^{2}-s^{2}}{2 r_{0} r}\right)^{2 q_{f}}\left(x \hat{x}+\left(y-y_{f}\right) \hat{y}+\left(-z_{f}\right) \hat{z}\right)
$$

Hence, the numerator in equation (5) can be calculated numerically: 


$$
\begin{aligned}
I_{n} & =\int_{-D / 2}^{D / 2} \frac{\sqrt{(D / 2)^{2}-x^{2}}}{\sqrt{(D / 2)^{2}-x^{2}}}\left(\frac{1}{r^{3}}\left(\frac{r_{0}^{2}+r^{2}-s^{2}}{2 r_{0} r}\right)^{2 q_{f}}\left(\left(x \hat{x}+\left(y-y_{f}\right) \hat{y}+\left(-z_{f}\right) \hat{z}\right)\right)\right) \square(-\hat{z}) d x d y \\
& =\int_{-D / 2}^{D / 2} \int_{(D / 2)^{2}-x^{2}} \frac{z_{f}\left(x_{0}^{2}+\left(y_{0}-y_{f}\right)^{2}+x^{2}+\left(y-y_{f}\right)^{2}+2 z_{f}^{2}-\left(x-x_{0}\right)^{2}-\left(y-y_{0}\right)^{2}\right)^{2 q_{f}}}{\left(2 \sqrt{x_{0}^{2}+\left(y_{0}-y_{f}\right)^{2}+z_{f}^{2}}\right)^{2 q_{f}}\left(\sqrt{x^{2}+\left(y-y_{f}\right)^{2}+z_{f}^{2}}\right)^{2 q_{f}+3}} d x d y
\end{aligned}
$$

Finally, the spillover efficiency is obtained as:

$$
\eta_{s}=\frac{I_{n}}{I_{d}}=\eta_{s}\left(D, y_{f}, z_{f}, q_{f}, x_{0}, y_{0}\right)
$$

From equation (10), we can see that $\eta_{s}$ is independent of $q_{e}$.

\subsubsection{Comparison between New Method and Original Method}

To verify the performance of the new method, a special case where the surface $\sigma$ is a simple conical surface, a central feed reflectarray with a circular aperture, is considered as the integral of equation (4) can be calculated analytically. For this case, the analytical result can be obtain [1]:

$$
\eta_{s}=1-(\cos \alpha)^{2 q_{f}+1}
$$

where the angle $\alpha$ is used to represent the half aperture angle of the cone.

As $y_{f}=0, z_{f}=280 \mathrm{~mm}$ and $q_{f}=6.5$, the diameter of the reflectarray aperture D is varying with $\alpha$.The comparison between new method and original method is shown in Figure 3. The results from the new and original method agree very well with each other.

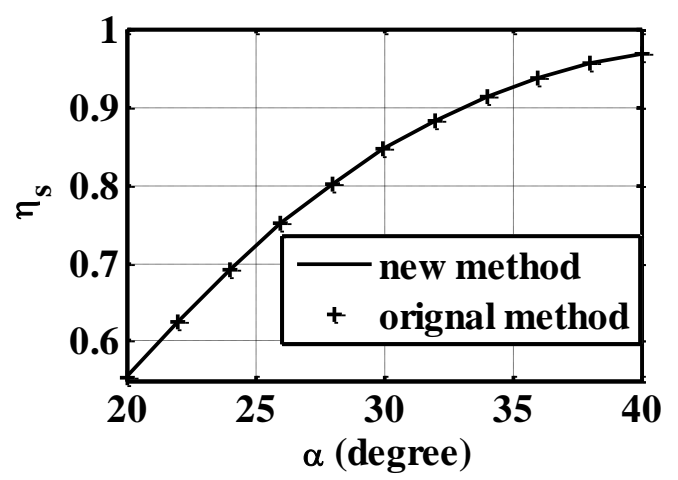

Figure 3. Comparison between New Method and Original Method

\subsubsection{The Taper Efficiency}

For the reflectarray antennas, the taper efficiency is defined to express the gain loss caused by the aperture amplitude distribution [4]. The evaluation of $\eta_{s}$ is through the following equation: 


$$
\eta_{t}=\frac{1}{A_{a}} \frac{\left|\iint_{A} I(x, y) d A\right|^{2}}{\iint|I(x, y)|^{2} d A}
$$

Where $I(x, y)$ is the aperture amplitude distribution with the purely certain polarized field. For the configuration in Figure 1, it can be obtained by using the given pattern model of the feed horn and reflectarray element. The expression of $I(x, y)$ is as follow:

$$
I(x, y)=\frac{\left(\cos \theta_{f}\right)^{q_{f}}\left(\cos \theta_{e}\right)^{q_{e}}}{r}
$$

Here, by applying the geometric parameters in Table $1, I(x, y)$ can be rewritten in the Cartesian coordinates, as follow:

$$
\begin{aligned}
I(x, y) & =\frac{1}{r}\left(\frac{r_{0}^{2}+r^{2}-s^{2}}{2 r_{0} r}\right)\left(\frac{z_{f}}{r}\right)^{q_{e}} \\
& =\frac{z_{f}^{q_{e}}\left(x_{0}^{2}+\left(y_{0}-y_{f}\right)^{2}+x^{2}+\left(y-y_{f}\right)^{2}+2 z_{f}^{2}-\left(x-x_{0}\right)^{2}-\left(y-y_{0}\right)^{2}\right)^{q_{f}}}{\left(2 \sqrt{x_{0}^{2}+\left(y_{0}-y_{f}\right)^{2}+z_{f}^{2}}\right)^{q_{f}}\left(\sqrt{x^{2}+\left(y-y_{f}\right)^{2}+z_{f}^{2}}\right)^{q_{f}+q_{e}+1}}
\end{aligned}
$$

For a circular aperture, the taper efficiency can be calculated numerically as follow:

$$
\eta_{t}=\frac{4}{\pi D^{2}} \frac{\left\{\int_{-D / 2} \int_{D / 2}^{D / 2 \sqrt{(D / 2)^{2}-x^{2}}} \frac{\left(x_{0}^{2}+\left(y_{0}-y_{f}\right)^{2}+x^{2}+\left(y-y_{f}\right)^{2}+2 z_{f}^{2}-\left(x-x_{0}\right)^{2}-\left(y-y_{0}\right)^{2}\right)^{q_{f}}}{\left(2 \sqrt{x_{0}^{2}+\left(y_{0}-y_{f}\right)^{2}+z_{f}^{2}}\right)^{q_{f}}\left(\sqrt{x^{2}+\left(y-y_{f}\right)^{2}+z_{f}^{2}}\right)^{q_{f}+q_{c}+1}} d x d y \mid\right\rceil^{2} \mid}{\int_{-D / 2} \int_{-\sqrt{(D / 2)^{2}-x^{2}}} \frac{\left(x_{0}^{2}+\left(y_{0}-y_{f}\right)^{2}+x^{2}+\left(y-y_{f}\right)^{2}+2 z_{f}^{2}-\left(x-x_{0}\right)^{2}-\left(y-y_{0}\right)^{2}\right)^{2 q_{f}}}{\left(2 \sqrt{x_{0}^{2}+\left(y_{0}-y_{f}\right)^{2}+z_{f}^{2}}\right)^{2 q_{f}}\left(\sqrt{x^{2}+\left(y-y_{f}\right)^{2}+z_{f}^{2}}\right)^{2\left(q_{f}+q_{c}+1\right)}} d x d y}
$$

Equation (13) shows that the taper efficiency is related to seven parameters, as follow:

$$
\eta_{t}=\eta_{t}\left(D, y_{f}, z_{f}, q_{f}, x_{0}, y_{0}, q_{e}\right)
$$

\subsection{Derivation of Radiation Patterns}

Most reflectarray analysis approaches approximate the reflectarray elements on the aperture plane as identical elements that constitute an array, then, the reflect characteristic of the element is usually obtained with a full wave simulation by using an infinite-array approach. Array theory method or aperture field method is then applied to calculate the radiation patterns of the reflectarray antennas.

\subsubsection{Array Theory Method}

The radiation patterns of the reflectarray with $N x \times N y$ elements shown in Figure 1 can be related to the radiation patterns of a two dimensional planar array as follow: 


$$
E(\theta, \phi)=\sum_{m=1}^{N x} \sum_{n=1}^{N y} A(\theta, \phi) I(m, n) \mathrm{e}^{\left(j k\left(\vec{d}_{m n} \square \hat{u}\right)\right)}
$$

Where $\vec{d}_{m n} \square \hat{u}=x_{m n} \sin \theta \cos \phi+y_{m n} \sin \theta \sin \phi, A(\theta, \phi)$ is the element patterns, $I(m, n)$ is the element excitation vector.

With the given pattern model of the element, $A(\theta, \phi)$ can be expressed as follow:

$$
A(\theta, \phi)=(\cos \theta)^{q_{e}}
$$

As we know, the amplitude of the element excitation $I(m, n)$ has been obtained from equation (12). For reflectarray antennas, the phase of the element excitation $I(m, n)$ includes two parts: the spatial delay $-k r_{m n}$ of the feed horn caused by the Euclidian distance between the feed horn and the $m n t h$ element, the required phased delay $\varphi_{m n}$ of the $m n t h$ element designed to set the main beam in $\hat{u}_{0}$ direction. Such that, the complex element excitation can be written as follow:

$$
I(m, n)=\frac{\left(\cos \theta_{f}(m, n)\right)^{q_{f}}\left(\cos \theta_{e}(m, n)\right)^{q_{e}}}{r_{m n}} \mathrm{e}^{j\left(-k r_{m n}+\varphi_{m n}\right)}
$$

Where the term $\varphi_{m n}$ includes two parts: the spatial delay compensation and the element phase shift to make the main beam point to $\hat{u}_{0}$ direction.

With these derivations, the radiation patterns of the reflectarray antennas can be expressed in this form:

$$
E(\theta, \phi)=(\cos \theta)^{q_{e}} \sum_{m=1}^{N x} \sum_{n=1}^{N y} \frac{\left(\cos \theta_{f}(m, n)\right)^{q_{f}}\left(\cos \theta_{e}(m, n)\right)^{q_{e}}}{r_{m n}} \mathrm{e}^{j\left(-k r_{m n}+\varphi_{m n}\right)} \mathrm{e}^{j k\left(x_{m n} \sin \theta \cos \phi+y_{m n} \sin \theta \sin \phi\right)}
$$

\subsubsection{Aperture Field Method}

The radiation pattern of an ideal polarized feed horn with a fixed phase center can be expressed as follow:

$$
\left.\vec{E}\left(\theta^{\prime}, \phi^{\prime}\right)=\frac{e^{-j k r}}{r}\left[\hat{\theta}^{\prime} U_{E}\left(\theta^{\prime}\right)\left(a e^{j \psi} \cos \phi^{\prime}+b \sin \phi^{\prime}\right)+\hat{\phi}^{\prime} U_{H}\left(\theta^{\prime}\right)\left(-a e^{j \psi} \sin \phi^{\prime}\right)+b \cos \phi^{\prime}\right)\right]
$$

Where $U_{E}\left(\theta^{\prime}\right)$ and $U_{H}\left(\theta^{\prime}\right)$ are the E- and H-plane radiation patterns of the horn antenna, respectively, which can be express as $U_{E}\left(\theta^{\prime}\right)=U_{H}\left(\theta^{\prime}\right)=\left(\cos \theta^{\prime}\right)^{q_{f}}$ [7]. By choosing $(a, b, \psi)$, any polarized feed can be presented [8].

First, the incident fields on the reflectarray aperture are obtained from the polarized field of the feed horn using the matrix transformations from the feed coordinates $\left\{\hat{c}^{\prime}\right\}$ to the reflectarray coordinates $\{\hat{c}\}$ [9]. Note that, for most reflectarray elements, the amplitude of the reflect coefficient is very close to unity [10]. As a consequence, the amplitude of incident and reflected fields on the element can be set equally. Then, the aperture field is expressed in Cartesian components as follow:

$$
\vec{E}_{a}(x, y)=E_{x}(x, y) \hat{x}+E_{y}(x, y) \hat{y}
$$


The far electric field of reflectarray is obtained from the aperture fields using the angular spectrum of plane waves and a stationary phase approximation [4].The far electric field with the dependence on $R$ suppressed can be written as follow:

$$
\vec{E}(\theta, \phi)=\frac{j k}{2 \pi}\left[(\hat{\theta} \cos \phi-\hat{\phi} \sin \phi \cos \theta) \tilde{E}_{x}+(\hat{\theta} \sin \phi+\hat{\phi} \cos \phi \cos \theta) \tilde{E}_{y}\right]
$$

Where $\tilde{E}_{x / y}$ is the 2D Fourier transforms of the complex $E_{x / y}$, given by:

$$
\tilde{E}_{x / y}(\theta, \phi)=\iint_{A} E_{x / y}(x, y) e^{j k(x \sin \theta \cos \phi+y \sin \theta \sin \phi)}
$$

Assuming that the reflect field on mnth element is constant complex $E_{x / y}(m, n)$ with amplitude of $A_{x / y}(m, n)$ and phase of $\varphi_{x / y}(m, n)$. Where the double integral is simplified as a double sum:

$$
\tilde{E}_{x / y}(\theta, \phi)=B \sum_{m=1}^{N x} \sum_{n=1}^{N y} A_{x / y}(m, n) e^{j \varphi_{x / y}(m, n)} e^{j k\left(x_{m n} \sin \theta \cos \phi+y_{m n} \sin \theta \sin \phi\right)}
$$

With $B=p^{2} \operatorname{sinc}(0.5 \mathrm{kp} \sin \theta \cos \phi) \operatorname{sinc}(0.5 \mathrm{kp} \sin \theta \sin \phi), \quad p$ is the unit-cell periodicity.

From our calculation, the situation that the $x$-component of the incident $y^{\prime}$ polarization is far less than the y-component is found, and same situation of $x^{\prime}$ polarization is also found. In order to simplify the computation of the radiation pattern, the $x$-component of the incident $y^{\prime}$ polarization and the $y$-component of the incident $x^{\prime}$ polarization are approximated to zeros. The aperture field for the incident $x^{\prime}$ and $y^{\prime}$ polarized field of feed horn can be rewritten as follow:

$$
\begin{aligned}
& \vec{E}_{a x^{\prime}}(x, y)=E_{x}(x, y) \hat{x} \\
& \vec{E}_{a y^{\prime}}(x, y)=E_{y}(x, y) \hat{y}
\end{aligned}
$$

Taking equation (25) into account and substituting equation (24) into equation (22), the far field expressions of the incident $x^{\prime}$ and $y^{\prime}$ polarized field of feed horn become:

$$
\begin{aligned}
& \vec{E}_{x^{\prime}}(\theta, \phi)=\frac{j k}{2 \pi}(\hat{\theta} \cos \phi-\hat{\phi} \sin \phi \cos \theta) B \sum_{m=1}^{N x} \sum_{n=1}^{N y} A_{x}(m, n) e^{j \varphi_{x}(m, n)} e^{j k\left(x_{m n} \sin \theta \cos \phi+y_{m n} \sin \theta \sin \phi\right)} \\
& \vec{E}_{y^{\prime}}(\theta, \phi)=\frac{j k}{2 \pi}(\hat{\theta} \sin \phi+\hat{\phi} \cos \phi \cos \theta) B \sum_{m=1}^{N x} \sum_{n=1}^{N y} A_{y}(m, n) e^{j \varphi_{y}(m, n)} e^{j k\left(x_{m n} \sin \theta \cos \phi+y_{m n} \sin \theta \sin \phi\right)}
\end{aligned}
$$

For general cases, only the co-polar radiation patterns are concerned for the application of reflectarray antennas. Hence, the co-polar radiation patterns are determined by means of Ludwig's third definition [11], the expressions are written as follow:

$$
\begin{aligned}
& \left|E_{c o x^{\prime}}\right|=\frac{k}{2 \pi}|B|\left|(\cos \phi)^{2}+\cos \theta(\sin \phi)^{2}\right|\left|\sum_{m=1}^{N x} \sum_{n=1}^{N y} A_{x}(m, n) e^{j \varphi_{x}(m, n)} e^{j k\left(x_{m n} \sin \theta \cos \phi+y_{m m} \sin \theta \sin \phi\right)}\right| \\
& \left|E_{c o y^{\prime}}\right|=\frac{k}{2 \pi}|B|\left|(\sin \phi)^{2}+\cos \theta(\cos \phi)^{2}\right|\left|\sum_{m=1}^{N x} \sum_{n=1}^{N y} A_{y}(m, n) e^{j \varphi_{y}(m, n)} e^{j k\left(x_{m n} \sin \theta \cos \phi+y_{m n} \sin \theta \sin \phi\right)}\right|
\end{aligned}
$$




\subsubsection{Computational Speedup Using Discrete Fourier Transform}

In the previous sections, it is shown that both approaches for radiation patterns calculation require the evaluation of a double summation. The double summation can be calculated by means of 2D inverse discrete Fourier transform (IDFT) [12]. To use 2D IDFT, the direction cosine $u=\sin \theta \cos \phi$ and $v=\sin \theta \sin \phi$ are introduced, and the discretization of $u$ and $v$ is defined as follow:

$$
\begin{aligned}
& u_{m}=\frac{2 \pi}{M k d}\left(m-\frac{M}{2}\right), m=0,1, \cdots, M-1 \\
& v_{n}=\frac{2 \pi}{N k d}\left(n-\frac{N}{2}\right), \quad n=0,1, \cdots, N-1
\end{aligned}
$$

Then, the equation (19) and (27) can be rewritten as follow:

$$
\begin{aligned}
& E(m, n)=M N\left(\sqrt{1-u_{m}^{2}-v_{n}^{2}}\right)^{q_{e}} \operatorname{IDFT}\left(\frac{\left(\cos \theta_{f}(m, n)\right)^{q_{f}}\left(\cos \theta_{e}(m, n)\right)^{q_{e}}}{r_{m n}} \mathrm{e}^{\left(j\left(-k r_{m n}+\varphi_{m n}\right)\right)}\right) \\
& \left|E_{c o x^{\prime}}(m, n)\right|=\frac{k}{2 \pi} M N\left|B^{\prime}\right|\left|C_{x}\right|\left|\operatorname{IDFT}\left(A_{x}(m, n) e^{j \varphi_{x}(m, n)}\right)\right| \\
& \left|E_{c o y^{\prime}}(m, n)\right|=\frac{k}{2 \pi} M N\left|B^{\prime}\right|\left|C_{y}\right|\left|\operatorname{IDFT}\left(A_{y}(m, n) e^{j \varphi_{y}(m, n)}\right)\right|
\end{aligned}
$$

With $B^{\prime}(m, n)=p^{2} \operatorname{sinc}\left(0.5 k p u_{m}\right) \operatorname{sinc}\left(0.5 \mathrm{kp} v_{n}\right)$

$$
C_{x}=1+\frac{v_{n}^{2}\left(\sqrt{1-u_{m}^{2}-v_{n}^{2}}-1\right)}{u_{m}^{2}+v_{n}^{2}}, C_{y}=1+\frac{v_{n}^{2}\left(\sqrt{1-u_{m}^{2}-v_{n}^{2}}-1\right)}{u_{m}^{2}+v_{n}^{2}}
$$

\section{Numerical Results}

\subsection{Parameters Study of Aperture Efficiency}

There are seven parameters $\left(D, q_{f}, q_{e}, y_{f}, z_{f}, x_{0}, y_{0}\right)$ effecting the aperture efficiency of a reflectarray configured in figure 1 . However, the first three parameters usually have been determined before aperture efficiency analysis, such as $D$ determined by the required directivity, $q_{f}$ determined by the chosen feed horn, $q_{e}$ determined by the chosen element. Only the feed position $\left(y_{f}, z_{f}\right)$ and feed beam point $\left(x_{0}, y_{0}\right)$ need to be analyzed for the influence to the aperture efficiency in a practical reflectarray design. To a circular aperture reflectarray, designed at frequency of $12.5 \mathrm{GHz}$, with $D=15 \lambda$ ( $\lambda$ is the wavelength of $12.5 \mathrm{GHz}), q_{f}=6.5, q_{e}=1$, the effects of these two groups of parameter with are presented by means of the Numerical Integration Toolbox [13] of Matlab. 


\subsubsection{Feed Position}

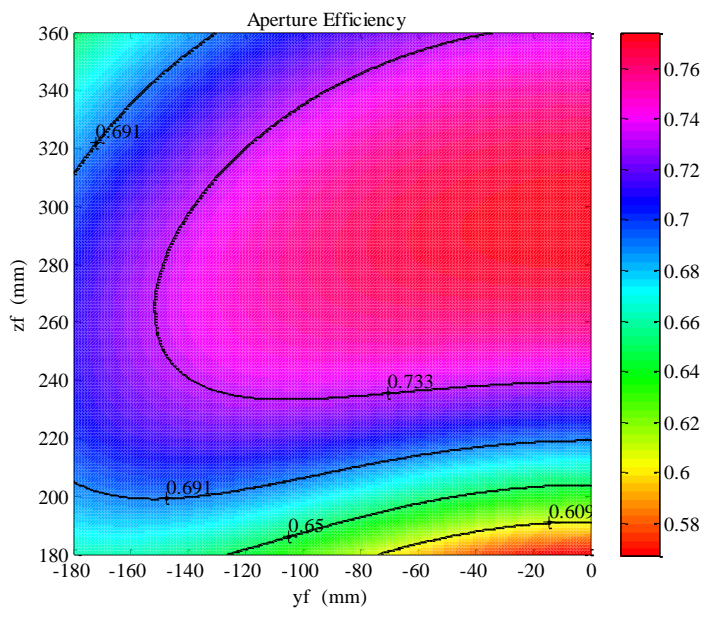

Figure 4. Aperture Efficiency vs. the Feed Position $\left(y_{f}, z_{f}\right)$

To analysis the effect of the feed position $\left(y_{f}, z_{f}\right)$, the feed beam point is fixed in $(0,0)$. The contoured view of the aperture efficiency variation associated to feed location is demonstrated in Figure 4. It is noted the aperture efficiency is varying slowly from $65 \%$ to $77 \%$. The maximum aperture efficiency is achieved near $z_{f}=280 \mathrm{~mm}$. When $z_{f}$ is fixed in $280 \mathrm{~mm}$, the aperture efficiency maintains almost constant until $y_{f}$ is shifted to $-100 \mathrm{~mm}$. Such that, for the offset feed case, $y_{f}$ can be shift to $-100 \mathrm{~mm}$ to avoid the blockage effect of feed horn and get maximum aperture efficiency at the same time.

\subsubsection{Feed Orientation}

The feed orientation is described by the feed beam point $P_{0}\left(x_{0}, y_{0}\right)$. Using the fixed feed position parameters: $y_{f}=-100 \mathrm{~mm}, z_{f}=280 \mathrm{~mm}$, the contoured aperture efficiency plot is shown in Figure 5. On this plot, the optimum aperture efficiency $77.2 \%$ is obtained when the feed beam point $P_{0}$ located in the origin. This optimum aperture efficiency point can be considered as the result of a compromise between the spillover efficiency and the taper efficiency. Besides, the symmetry of the aperture efficiency with respect to $x$-axes is observed.

Through the previous analysis, the feed position and orientation parameters for a reflectarray with $D=360 \mathrm{~mm}, q_{f}=6.5, q_{e}=1$, to obtain relative high aperture efficiency, are determined. 


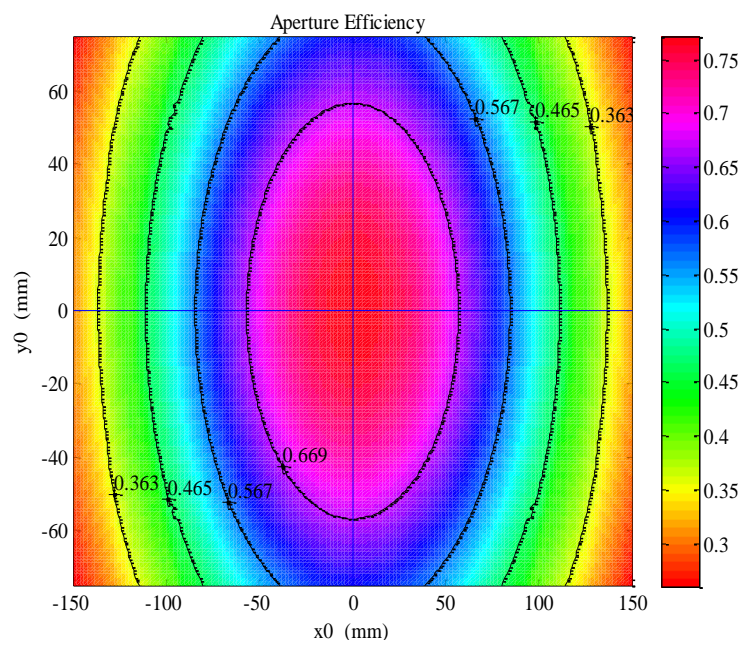

Figure 5. Aperture Efficiency vs. the Feed Orientation $\left(x_{0}, y_{0}\right)$

\subsection{Radiation Patterns}

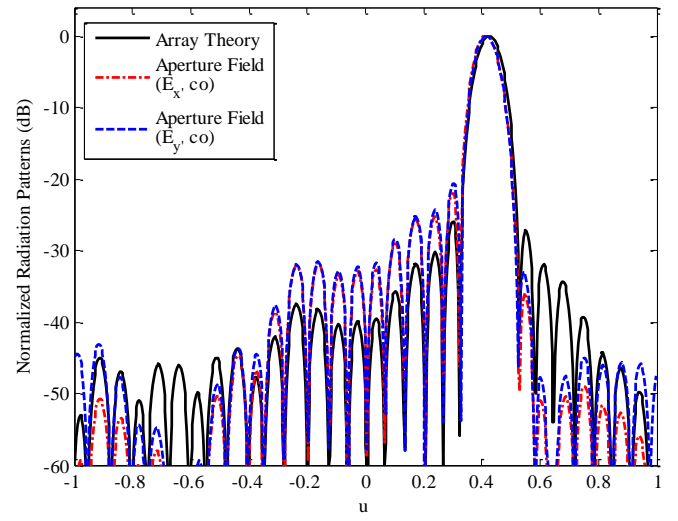

Figure 6. Normalized Radiation Patterns in $v=0$ Plane

The radiation patterns of the reflectarray antennas with the parameters obtained in the previous analysis are calculated by two approaches discussed in Section 2.2. The main beam direction $\hat{u}_{0}$ is set in the direction of $\left(\theta_{b}, \phi_{b}\right)=\left(25^{\circ}, 0^{\circ}\right)$. In the analysis of radiation patterns, the elements are considered as the ideal elements with the unit-cell periodicity of the element, $\lambda / 2$ at the frequency of $12.5 \mathrm{GHz}$, which can provide the required phase shift without phase error. The normalized radiation patterns in $v=0$ plane at $12.5 \mathrm{GHz}$ are demonstrated in Figure 6.

From the results presented in Figure 5 it could be seen that the calculated radiation patterns obtained by both methods were in close agreement with each other. In particular, the mainbeam directions, beam-widths, and general pattern shape are almost identical. However, a slight difference is observed in the side lobe region, caused by the approximation of polarized field adopt in the aperture field method. In comparison, the CPU times for the array-theory and aperture field approach are respectively $0.04 \mathrm{sec}$ and $3.5 \mathrm{sec}$ on the computer with eightcore $3.07 \mathrm{GHz}$ Intel Xeon W3550 and 6GB memory, which means that the array theory method is more suitable for a fast analysis of the reflectarray's radiation patterns without polarization consideration needed in the procedure of pattern optimization, while the aperture 
method is more suitable for the analysis of the reflectarray's radiation patterns with polarization consideration needed in the two polarized pattern optimization.

\section{Conclusion}

The estimation of the aperture efficiency and radiation patterns is necessary in the initial design of reflectarray antennas. This paper summarizes the approaches for the fast analysis of the aperture efficiency and radiation patterns of reflectarray antenna. The detail derivations of the aperture efficiency and radiation pattern associated with geometric parameters are performed in this paper. On the basis of those derivations, numerical results of a $\mathrm{Ku}$ band offset feed reflectarray are presented to show the parameters study for aperture efficiency, and compare two pattern analysis approaches performance. This paper provides the efficiency analysis tools for antenna engineers to evaluating the performance of the reflectarray they designed in the initial design stage.

\section{Acknowledgements}

This work is supported in part by the National Defense Pre-Research Foundation of China under Grant No.9140A01010412HK03004 and the Aerospace Innovation Fund under Grant No. HTCXJJKT-22.

\section{References}

[1] J. Huang and J. A. Encinar, "Reflect-array Antennas", John Wiley \& Son, Hoboken, New Jersey (2008).

[2] M. E. Biakowski and J. A. Encinar, "Reflect-array: Potentials and challenges", Proceeding of International Conference on Electromagnetics in Advanced Applications, (2007) September 17-21, Turin, Italy.

[3] D. M. Pozar, S. D. Targonski and H. D. Syrigos, IEEE, Transaction on Antennas and Propagation, vol. 2, no. 45, (1997).

[4] C. A. Balanis, "Antenna Theory Analysis and Design Third Edition", John Wiley \& Son, Hoboken, New Jersey, (2005).

[5] P. Nayeri, A. Z. Elsherbeni and F. Yang, IEEE, Antennas and Propagation Magazine, 1.55, (2013).

[6] A. Yu, F. Yang, A. Z. Elsherbeni, J. Huang and Y. R. Sami, "Microw", Opt. Tech. Lett. 2.52 (2010).

[7] Y. R. Sami, Cramer and P. Jr. Woo, IEEE, Transaction on Antennas and Propagation, 6.29, (1981).

[8] P. Lam, S. W. Lee, D. Chang and K. Lang, IEEE, Transaction on Antennas and Propagation. 6. 29, (1985).

[9] Y. R. Sami, IEEE, Transaction on Antennas and Propagation. 4.27, (1979).

[10] J. A. Zornoza and J. A. Encinar, International Journal of RF and Microwave Computer-Aided Engineering, 5.14, (2004).

[11] A. Ludwing, IEEE, Transaction on Antennas and Propagation. 1.21, (1973).

[12] W. P. M. N. Kelzer, IEEE Antennas and Propagation Magazine, 2.51, (2009).

[13] W. Howard and B. Gardner, "Numerical Integration Toolbox".

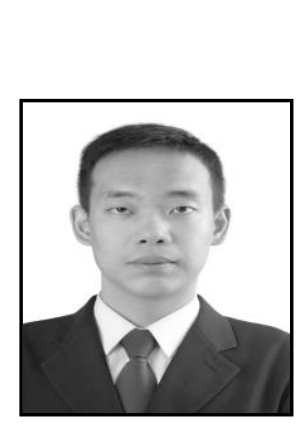

\section{Author}

Yan Qu, he obtained an MS in Electronic and Information Engineering from the school of Electronic and Information, Northwestern Polytechnical University, 2006. Then, he was recommended to a PhD in Electronic Science and Technology from the school of Electronic and Information, Northwestern Polytechnical University. His research interesting are array synthesis, design of Reflectarray. 